\title{
Recorrência da Crise Convulsiva após Terapia Anticonvulsivante com Sulfato de Magnésio em Pacientes com Eclâmpsia
}

\author{
Recurrence of Seizures after Anticonvulsant Therapy with Magnesium \\ Sulfate in Patients with Eclampsia \\ Melania Maria Ramos de Amorim, Luiz Carlos Santos, Ana Maria Feitosa Porto, \\ Leila Katz Dias Martins, Valdson Vieira
}

\begin{abstract}
RESUMO
Objetivos: determinar a freqüência de recorrência das crises convulsivas após tratamento com sulfato de magnésio, avaliando o tratamento adotado e o prognóstico materno.

Casuística e Métodos: analisaram-se todos os casos de eclâmpsia atendidos no IMIP entre janeiro de 1995 e junho de 1998. Sulfato de magnésio e oxigenoterapia foram administrados para todas as pacientes, interrompendo-se a gravidez após estabilização do quadro clínico. Determinou-se a freqüência de complicações maternas de acordo com a presença ou não de recorrência da crise convulsiva, utilizando-se o teste $\chi^{2}$ de associação, a um nivel de significância de $5 \%$.

Resultados: doze pacientes apresentaram recorrência da eclâmpsia após sulfato de magnésio (10\%), repetindo-se então metade da dose de ataque. Em 4 destas verificou-se nova recorrência, administrando-se então diazepam endovenoso. Depois do diazepam, uma paciente ainda teve crises repetidas, sendo então realizada infusão de fenitoina e, posteriormente, indução do coma barbitúrico (tionembutal). Essa paciente foi submetida a tomografia computadorizada, constatando-se hemorragia intracraniana. As complicações maternas foram significativamente mais freqüentes no grupo com recorrência: coma (16,7\% versus 0,9\%), acidose (50\% versus 2,9\%), edema agudo de pulmão (16,7\% versus 2,9\%), hemorragia cerebral (16,7\% versus $0 \%$ ) e insuficiencia renal aguda (16,7\% versus $1,9 \%)$. Ocorreram 3 casos de morte materna no grupo com recorrência (25\%) e 2 no grupo sem recorrência (1,9\%). Conclusões: a recorrência da crise convulsiva é pouco freqüente após uso do sulfato de magnésio (10\%), porém associa-se a aumento da morbimortalidade materna, requerendo acompanhamento em UTI e realização de tomografia para exclusão de hemorragia cerebral.
\end{abstract}

PALAVRAS-CHAVE: Eclâmpsia. Sulfato de magnésio. Eclâmpsia: recorrência. Morte materna.

\section{Introdução}

A eclâmpsia constitui uma das principais causas de morte materna em todo o mundo, representando uma situação de extrema emergência, com risco iminente de vida, que requer imediatos cuidados intensivos ${ }^{1-7}$. A preocupação inicial deve ser garantir oxigenação adequada e pro-

Instituto Materno-Infantil de Pernambuco (IMIP) - Centro de Atenção à Mulher (CAM)

Correspondência: Melania Maria Ramos de Amorim

IMIP - Coordenação de Ensino

Rua dos Coelhos, 300 - Boa Vista

50070-550 - Recife - PE

e-mail:melania@interway.com.br videnciar o controle das crises convulsivas, medidas básicas de suporte à vida ${ }^{8-11}$. Depois da estabilização do quadro clínico, está indicada, via de regra, a interrupção da gravidez, pois a única "cura" possivel para o processo mórbido desencadeado pelo vasoespasmo generalizado é o parto, com a expulsão do vilo corial do organismo materno ${ }^{10}$.

$\mathrm{O}$ sulfato de magnésio $\left(\mathrm{MgSO}_{4}\right)$ é, indubitavelmente, a droga ideal para o controle das convulsões eclâmpticas ${ }^{7,12-14}$. Utilizado empiricamente com esta finalidade desde $1925^{2}$, teve sua indicação questionada por algum tempo, sobretudo pelos neurologistas ${ }^{14}$, porém na década atual numerosas evidências vêm se acumulando que demonstram sua eficácia e segurança no tratamento 
da eclâmpsia ${ }^{12,13,15}$. Em 1995, o estudo do "Collaborative Eclampsia Trial", publicado no Lancet, comparou o uso do sulfato de magnésio com a fenitoína e o diazepam, verificando nítida superioridade do primeiro, com menor taxa de recorrência $(9,7 \%$ versus $17,1 \%$ e $27,9 \%$, respectivamente) e uma significativa redução da mortalidade materna (em torno de $50 \%)^{15}$.

Apesar da utilização correta da terapia anticonvulsivante, espera-se uma taxa de recorrência em torno de $10-20 \%$. Sibai ${ }^{6,16}$, tentando reduzir esta freqüência, propôs a administração de doses maiores de sulfato de magnésio (infusão de $2 \mathrm{~g}$ /hora), mas ainda encontrou uma taxa de recorrência de $16,7 \%$. Por outro lado, com a utilização de $1 \mathrm{~g} /$ hora, essa taxa foi aproximadamente $10 \%$ no estudo do "Collaborative Eclampsia Trial"15. Na verdade, os casos não-complicados de eclâmpsia respondem muito bem ao tratamento com sulfato de magnésio ${ }^{13,16}$, independente do regime administrado, e a recorrência da crise convulsiva deve sugerir a possibilidade de outros distúrbios neurológicos e, principalmente, de hemorragia intracraniana ${ }^{17,18}$.

Diversas modalidades terapêuticas têm sido propostas para a recorrência ${ }^{2,8,11,17}$, porém a morbimortalidade materna sofre considerável acréscimo quando as convulsões persistem, independente do tratamento ministrado, sendo freqüentes complicações como acidose, coma e edema agudo de pulmão ${ }^{2,17}$. A morte pode ocorrer subitamente em decorrência de hemorragia cerebral volumosa ou no curso da evolução clínica como corolário das outras complicações ${ }^{2,6,17}$.

Embora diversos estudos tenham sido publicados acerca do prognóstico materno na eclâmpsia, poucos tentam determinar o risco associado à recorrência da crise convulsiva, relatando-se em geral apenas sua freqüência, sem avaliar sua magnitude e quantificar o risco ${ }^{6,17,19-21}$.

Resolvemos portanto realizar o presente estudo com os objetivos de: 1) determinar a freqüência de recorrência da eclâmpsia após terapia anticonvulsivante com sulfato de magnésio; 2) avaliar o tratamento adotado na recorrência e seus resultados; 3) comparar as características clínicas e o prognóstico materno de acordo com a recorrência ou não da crise convulsiva.

\section{Pacientes e Métodos}

Desde janeiro de 1995 até junho de 1998 todos os casos de eclâmpsia atendidos no Centro de Atenção à Mulher do IMIP têm sido acompanhados prospectivamente, constituindo uma coorte que, até o presente, já conta com 120 pacientes (correspondendo a $0,6 \%$ do total de partos assistidos neste período). Este estudo foi aprovado pelo Comitê de Ética em Pesquisa de nossa instituição. Todas as pacientes envolvidas foram submetidas ao protocolo de conduta vigente no serviço desde 1988, que inclui medidas de emergência como oxigenoterapia e administração endovenosa de sulfato de magnésio pelo esquema de Zuspan $(6 \mathrm{~g}$, iv) ${ }^{22}$, além da interrupção da gravidez após estabilização do quadro clínico (a via de parto depende das condições cervicais e da vitalidade fetal) ${ }^{23}$.

De acordo com esse protocolo, a infusão endovenosa de sulfato de magnésio (1 g/hora) é mantida por 24 horas após o parto ou após a última crise convulsiva, salvo se vigentes manifestações de toxicidade. Os critérios para interrupção do sulfato de magnésio são: oligúria (até reinício da diurese), depressão respiratória e abolição dos reflexos tendinosos profundos. Reiniciase a droga após normalização dos parâmetros alterados. A hidralazina (5-20 mg iv) é utilizada para tratamento da crise hipertensiva (pressão diastólica maior ou igual que $110 \mathrm{mmHg}$ ).

Caso se verifique recorrência da crise convulsiva após sulfato de magnésio, aplica-se metade da dose de ataque ( $3 \mathrm{~g}$ iv) e, persistindo a crise convulsiva, administra-se diazepam iv (5-10 mg). Não cessando as crises convulsivas mesmo com esse esquema, indica-se administração de fenitoína $(15 \mathrm{mg} / \mathrm{kg})$ ou indução do coma barbitúrico (tionembutal) em unidade de terapia intensiva ${ }^{23}$, investigando-se nesses casos hemorragia cerebral como causa das convulsões (por meio de tomografia computadorizada e/ou ressonância magnética).

As pacientes desta coorte foram avaliadas no presente estudo de acordo com a resposta terapêutica ao sulfato de magnésio, verificando-se a freqüência de recorrência da crise convulsiva e o tratamento farmacológico empregado nesta situação. A resposta ao tratamento anticonvulsivante foi considerada satisfatória quando, além do término da crise convulsiva naquele instante, não se observaram mais convulsões durante todo o internamento.

Determinou-se a ocasião em que se manifestou a recorrência, de acordo com a fase do ciclo gravidico-puerperal (pré-parto, intraparto ou pósparto) e a fase de administração do sulfato de magnésio (ataque ou manutenção). Avaliou-se também o intervalo em horas transcorrido entre a administração da dose de ataque e nova crise convulsiva, e entre o parto e nova crise convulsiva. Compararam-se as características clínicas e laboratoriais das pacientes, considerando-se estas como variáveis independentes e a recorrência como variável dependente. A seguir, avaliou- 
se o prognóstico materno em função da presença ou não de recorrência, que nesta etapa passou ser tratada como variável independente, representando as complicações maternas e o óbito as variáveis dependentes.

Para as finalidades deste estudo, definiu-se como eclâmpsia a ocorrência de convulsões tônico-clônicas, generalizadas, em paciente com hipertensão induzida pela gestação ${ }^{9}$. Considerou-se hipertensão induzida pela gestação o diagnóstico de hipertensão (PAS $\geq 140 \mathrm{mmHg}$ e/ou PAD $\geq 90$ $\mathrm{mmHg}$ ), proteinúria (1+ ou mais de proteinúria de fita - labstix - ou proteinúria de 24 horas a partir de $300 \mathrm{mg} / 1 / 24$ horas) e hiperuricemia (ácido úrico $>4,5 \mathrm{mg} \%$ ), com ou sem edema ${ }^{9,23,24}$. Denominou-se a eclâmpsia sobreposta quando esta se manifestou em paciente com diagnóstico de hipertensão crônica pré-existente ${ }^{9}$. Recorrência foi caracterizada caso persistissem convulsões após a administração da dose de ataque do sulfato de magnésio, ou se estas voltassem a ocorrer na vigência da manutenção desta droga.

As características clínicas maternas estudadas foram: idade (em anos completos), paridade (número de partos anteriores), idade gestacional (em semanas) e níveis tensionais (pressão arterial sistólica e diastólica) na admissão. A idade gestacional foi determinada pela data da última menstruação, quando esta era conhecida e confiável (história de ciclos regulares), ou pela ultrasonografia, quando não era disponivel a data da última menstruação. A partir de 32 semanas, considerou-se a idade gestacional calculada pelo método de Capurro realizado após o nascimento. Os niveis tensionais foram verificados com a paciente deitada no leito, utilizando-se as normas propostas pela "American Heart Association".

Em relação às características laboratoriais, estudaram-se os valores de uréia, creatinina, ácido úrico, plaquetas, transaminases e bilirrubinas, bem como a proteinúria de fita (labstix), exames colhidos logo após o tratamento anticonvulsivante e a estabilização clínica das pacientes.

Avaliou-se a presença das seguintes complicações maternas: sindrome HELLP, descolamento prematuro de placenta, edema agudo de pulmão, insuficiência renal aguda, coagulação intravascular disseminada, acidose, hemorragia digestiva, sepse, insuficiência respiratória, hemorragia cerebral, coma, choque e óbito.

Síndrome HELLP foi definida pelos critérios de $\mathrm{Sibai}^{25}$, incluindo plaquetopenia $(<100.000 /$ $\mathrm{mm}^{3}$ ), aumento de TGO (>70 U Frankel) e de bilirrubinas (bilirrubina total $>1,2 \mathrm{mg} \%$ ) e esfregaço anormal (esquisócitos, poiquilócitos) do sangue periférico, sendo considerada completa quando todos os parâmetros encontravam-se alterados e incom- pleta na presença de apenas 1 ou 2 dos parâmetros anormais. Considerou-se edema agudo de pulmão a presença de taquidispnéia, cianose, taquicardia e estertores crepitantes. Diagnosticou-se insuficiência renal aguda pela evidência de oligoanúria (<400 ml/24 horas) em associação a niveis elevados de uréia e creatinina. Coagulação intravascular disseminada (CIVD) foi definida pela presença de manifestações hemorrágicas (equimoses, petéquias, gengivorragia, sangramento genital, sangramento pelos locais de punção ou ferida operatória) e alteração das provas de coagulação. Um ou mais episódios de hematêmese caracterizaram a hemorragia digestiva. Hemorragia cerebral foi diagnosticada por tomografia computadorizada e/ ou ressonância magnética (realizada apenas em pacientes com suspeita clínica).

A análise estatística foi realizada no programa de domínio público Epi-Info 6.04b, utilizandose os testes $\chi^{2}$ de associação e exato de Fisher para a comparação de variáveis categóricas e o teste não-paramétrico de Mann-Whitney para as variáveis numéricas, a um nivel de significância de $5 \%$. Calculou-se a razão de risco (RR) e seu intervalo de confiança a 95\%, para determinação do risco relativo de morte materna de acordo com a presença ou não de recorrência.

\section{Resultados}

Verificou-se recorrência das convulsões em $12(10 \%)$ das 120 pacientes com eclâmpsia submetidas à terapia anticonvulsivante com sulfato de magnésio. Entre os casos de recorrência, 3 (25\%) pacientes tiveram nova crise convulsiva logo após a dose de ataque, ao passo que 9 (75\%) só vieram a apresentar recorrência durante a fase de manutenção do sulfato de magnésio. Em relação à fase do ciclo gravídico-puerperal, $3(25 \%)$ tiveram recorrência anteparto, $1(8,3 \%)$ durante o parto (periodo expulsivo) e 9 (75\%) no pós-parto, sendo que uma destas já tinha apresentado recorrência logo após a dose de ataque do sulfato de magnésio, ainda no período anteparto. O intervalo médio entre a administração da dose de ataque e a recorrência e entre o parto e a recorrência foram de, respectivamente, 6,5 e 8,0 horas (Tabela 1).

Quando se analisou o tratamento adotado na presença de nova crise convulsiva, encontrou-se que todas as pacientes receberam metade da dose de ataque do sulfato de magnésio ( $3 \mathrm{~g})$, conforme o protocolo do serviço, obtendo-se resposta satisfatória (ausência de novas convulsões) em 8 destas (67\%). Não houve efeitos deletérios (abolição dos reflexos ou parada respiratória) seguindo a admi- 
nistração dessa dose. Quatro pacientes (33\%) voltaram a apresentar crise convulsiva depois desta dose, e todas receberam injeção endovenosa de diazepam. Após o diazepam, apenas 1 paciente voltou a ter convulsões, requerendo o uso de fenitoína por via endovenosa. Ainda assim, persistiram novas crises, desencadeando-se o coma. Nesta paciente realizou-se tomografia computadorizada, diagnosticando-se hemorragia intracraniana, e indicou-se a indução de coma barbitúrico com tionembutal (Tabela 2). No entanto, constatou-se o óbito cerca de 22 horas depois de sua admissão no IMIP.

Tabela 1 - Ocasião da recorrência da crise convulsiva após o uso do sulfato de magnésio.

\begin{tabular}{|c|c|c|}
\hline Ocasião da recorrência & $\mathbf{n}$ & $\%$ \\
\hline \multicolumn{3}{|l|}{$\begin{array}{l}\text { Em relação à administração do sulfato } \\
\text { de magnésio }\end{array}$} \\
\hline $\begin{array}{l}\text { Imediatamente após a dose de } \\
\text { ataque }\end{array}$ & 3 & 25,0 \\
\hline Durante a fase de manutenção & 9 & 75,0 \\
\hline \multicolumn{3}{|l|}{$\begin{array}{l}\text { Intervalo (horas) entre a dose de } \\
\text { ataque e nova crise convulsiva }\end{array}$} \\
\hline Variação & $0-18$ & \\
\hline $\bar{x} / D P$ & $6,5 / 6,1$ & \\
\hline \multicolumn{3}{|l|}{ Em relação ao parto } \\
\hline Anteparto & 3 & 25,0 \\
\hline Intraparto & 1 & 8,3 \\
\hline Pós-parto & $9^{*}$ & 75,0 \\
\hline \multicolumn{3}{|l|}{$\begin{array}{l}\text { Intervalo (horas) entre o parto e a } \\
\text { nova crise convulsiva (X/DP) }\end{array}$} \\
\hline Variação & $1-14$ & \\
\hline $\bar{x} / D P$ & $8,0 / 4,3$ & \\
\hline Total de casos de recorrência & 12 & 100,0 \\
\hline
\end{tabular}

Tabela 2 - Tratamento e resposta terapêutica da recorrência da crise convulsiva em pacientes com eclâmpsia.

\begin{tabular}{|c|c|c|c|}
\hline $\begin{array}{l}\text { Tratamento da } \\
\text { recorrência }\end{array}$ & $\mathrm{n}(\%)$ * & $\begin{array}{c}\text { Cessaram as } \\
\text { Sim (\%) }\end{array}$ & $\begin{array}{c}\text { convulsões } \\
\text { Não (\%) }\end{array}$ \\
\hline $\begin{array}{l}\text { Repetição de } 1 / 2 \text { da } \\
\text { dose de ataque do } \\
\text { sulfato de magnésio }\end{array}$ & $12(100,0)$ & $8(66,7)$ & $4(33,3)$ \\
\hline Diazepam ev & $4 \quad(33,3)$ & $3(75,0)$ & $1(25,0)$ \\
\hline Fenitoína ** & $(8,3)$ & - & $1(100,0)$ \\
\hline Coma barbitúrico ** & $(8,3)$ & $1(100,0)$ & - \\
\hline $\begin{array}{l}\text { Total de casos de } \\
\text { recorrência }\end{array}$ & $12(100,0)$ & & \\
\hline
\end{tabular}

\footnotetext{
* Uma paciente pode estar incluída em uma ou mais opções de tratamento, de acordo com a resposta terapêutica

** Paciente com diagnóstico de hemorragia intracraniana
}

Estudaram-se as características clinicas e obstétricas das pacientes com eclâmpsia, de acordo com a recorrência ou não da crise convulsiva (Tabela 3), não se encontrando diferença estatisticamente significante entre idade, paridade, idade gestacional e niveis tensionais na admissão entre os 2 grupos. Verificou-se o predominio de pacientes jovens (média de 25 anos), primíparas (77\%), com idade gestacional em torno de 35 semanas, enquanto os niveis tensionais foram de aproximadamente $170 \times 115 \mathrm{mmHg}$. A freqüência de pacientes com hipertensão arterial sistêmica crônica (eclâmpsia sobreposta) foi contudo significativamente mais elevada no grupo que apresentou recorrência $(33 \%)$ em relação ao grupo sem recorrência $(9 \%)$.

Tabela 3 - Características clínicas e obstétricas das pacientes com eclâmpsia de acordo com a presença ou não de recorrência.

\begin{tabular}{|c|c|c|c|}
\hline Característica & $\begin{array}{l}\text { Com recorrência } \\
\qquad n=12\end{array}$ & $\begin{array}{l}\text { Sem recorrência } \\
\qquad n=108\end{array}$ & $p$ \\
\hline Idade (x/DP) & $25,6 / 3,8$ & $24,8 / 2,6$ & 0,4 \\
\hline Paridade (mediana) & 0 & 0 & 0,12 \\
\hline Primíparas $(\%, n)$ & $66,7 \%$ (8 casos) & 77,8 (84 casos) & 0,29 \\
\hline $\begin{array}{l}\text { Idade gestacional } \\
(\bar{x} / D P)\end{array}$ & $34,6 / 1,6$ & $35,2 / 1,2$ & 0,11 \\
\hline $\begin{array}{l}\text { PAS na admissão } \\
(\bar{x} / D P)\end{array}$ & 169,6 / 22,4 & 165,2 / 12,6 & 0,43 \\
\hline $\begin{array}{l}\text { PAD na admissão } \\
(\bar{x} / D P)\end{array}$ & 116,2 / 10,8 & $114,8 / 9,7$ & 0,63 \\
\hline $\begin{array}{l}\text { HAS crônica associada } \\
(\%, n)\end{array}$ & $33,3 \%$ (4casos) & $9,3 \%$ (10 casos) & $0,03^{*}$ \\
\hline \multicolumn{4}{|c|}{$\begin{array}{l}\bar{x}=\text { Média, } D P=\text { desvio-padrão } \\
\text { PAS = Pressão arterial sistólica } \\
\text { PAD = Pressão arterial diastólica } \\
\text { HAS = Hipertensão arterial sistêmica } \\
\text { * Diferença estatisticamente significativa }(p<0,05)\end{array}$} \\
\hline
\end{tabular}

Apesar de os valores laboratoriais de uréia, creatinina e ácido úrico serem mais elevados entre as pacientes com recorrência (média de 46 $\mathrm{mg} \%, 0,9 \mathrm{mg} \%$ e $7,2 \mathrm{mg} \%$, respectivamente, versus $36 \mathrm{mg} \%, 0,7 \mathrm{mg} \%$ e 6,0 mg\%, valores médios para as pacientes sem recorrência), esta diferença só foi significativa para os valores de ácido úrico. A mediana de proteinúria de fita $(4+)$ foi igual nos 2 grupos.

Avaliando-se as complicações maternas associadas, todas foram mais freqüentes quando havia recorrência (Tabela 4): acidose metabólica (50\% versus $2,9 \%)$, sindrome HELLP (33,3\% versus $12,5 \%)$, coma $(16,7 \%$ versus $0,9 \%)$, edema agudo de pulmão $(16,7 \%$ versus $2,9 \%)$, insuficiência renal aguda (16,7\% versus $1,9 \%)$ e hemorragia intracraniana (encontrados 2 casos, apenas entre os 
casos com recorrência, 16,7\%), embora não tenha sido encontrada significância estatística para a diferença na freqüência de síndrome HELLP e edema agudo de pulmão.

Tabela 4 - Complicações maternas em pacientes com eclâmpsia de acordo com a recorrência ou não de crise convulsiva.

\begin{tabular}{lccc}
\hline Complicações * & $\begin{array}{c}\text { Com recorrência } \\
\mathbf{n}=12(\%)\end{array}$ & $\begin{array}{c}\text { Sem recorrência } \\
\mathbf{n}=108(\%)\end{array}$ & $\mathbf{p}$ \\
\hline Acidose & $6(50,0)$ & $3(2,9)$ & $<0,0001$ \\
$\begin{array}{l}\text { Síndrome HELLP } \\
\text { (completa/incompleta) }\end{array}$ & $4(33,3)$ & $15(12,5)$ & 0,09 \\
$\begin{array}{l}\text { Coma } \\
\text { Edema agudo de }\end{array}$ & $2(16,7)$ & $1(0,9)$ & 0,03 \\
$\begin{array}{l}\text { pulmão } \\
\text { Insuficiência renal }\end{array}$ & $2(16,7)$ & $3(2,9)$ & 0,07 \\
$\begin{array}{l}\text { aguda } \\
\text { Hemorragia intra- }\end{array}$ & $2(16,7)$ & $2(1,9)$ & 0,05 \\
$\begin{array}{l}\text { craniana } \\
\text { Morte materna }\end{array}$ & $3(25,0)$ & $2(1,9)$ & 0,007 \\
\hline
\end{tabular}

* Uma paciente pode ter uma ou mais complicações

Ocorreram 3 casos de morte materna no grupo com recorrência ( $25 \%$ ) e 2 casos no grupo sem recorrência $(1,9 \%)$. O risco de morte materna foi 13,5 vezes maior na presença de recorrência, o que foi estatisticamente significativo, pois seu intervalo de confiança de $95 \%(2,5-72,9)$ não incluiu a unidade. As principais causas de morte materna no grupo com recorrência foram: coma (1 caso), hemorragia cerebral (1 caso) e edema agudo de pulmão (1 caso), ao passo que os 2 óbitos na ausência de recorrência deveram-se a choque hemorrágico (decorrente de coagulação intravascular disseminada) e embolia pulmonar.

\section{Discussão}

A taxa de recorrência de crises convulsivas após terapia com sulfato de magnésio no presente estudo foi de $10 \%$, correspondendo a 12 casos entre 120 pacientes com eclâmpsia atendidas no IMIP entre janeiro de 1995 e junho de 1998. A maioria destes casos, no entanto, respondeu satisfatoriamente à repetição de metade da dose de ataque do sulfato de magnésio: quase $70 \%$ das pacientes com recorrência não voltaram a apresentar novas convulsões depois de administrado esse esquema.

Esses resultados vêm dar apoio às conclusões de diversos autores que afirmam ser o sulfato de magnésio a droga ideal para o tratamento das convulsões eclâmpticas ${ }^{7,12,13,15}$. O percentual de recorrência aqui observado foi similar ao relatado no estudo do "Eclampsia Trial Collaborative Group" $(9,7 \%)^{15}$ e, portanto, nitidamente inferior às taxas encontradas quando se utilizou fenitoína ou diazepam ${ }^{15}$.

Embora alguns estudos preconizem utilizarse uma dose mais elevada de infusão endovenosa ( $2 \mathrm{~g} /$ hora na manutenção, ao invés de $1 \mathrm{~g} /$ hora $)^{16}$, para reduzir a taxa de recorrência, não acreditamos que esta seja necessária. O próprio Sibai ${ }^{6,7,16}$, que propôs esse esquema, encontrou em sua casuística em torno de $16 \%$ de recorrência, superior portanto ao nosso percentual e àquele encontrado pelo "Eclampsia Trial"15.

Parece que a recorrência associa-se muito mais a outros problemas neurológicos do que a uma eventual "falha" do sulfato de magnésio ${ }^{2,17,26}$. Estes distúrbios deveriam portanto ser sistematicamente investigados quando as convulsões persistem após a terapia anticonvulsivante com sulfato de magnésio. Estudos recentes com tomografia computadorizada e ressonância magnética têm evidenciado a presença de edema e/ou hemorragia cerebral associados à eclâmpsia ${ }^{26}$. Parece ocorrer na verdade uma extensa vasculopatia dentro do parênquima cerebral ${ }^{17,26}$. Diversos mecanismos têm sido propostos para explicar sua ocorrência, sendo o mais importante uma falha do mecanismo de auto-regulação do fluxo sanguíneo cerebral, levando ao edema e à hemorragia ${ }^{26}$. Sharf et al. ${ }^{27}$ atribuem a hemorragia cerebral à lesão arterial decorrente da vasoconstrição e isquemia, permitindo que os eritrócitos ultrapassem o compartimento vascular, porém é provável que as alterações da coagulação também estejam associadas, sobretudo nas grandes hemorragias ${ }^{2,26}$.

Encontramos na presente série 2 casos de hemorragia cerebral $(16,7 \%)$ entre as pacientes com recorrência, porém temos de salientar que a tomografia computadorizada só foi realizada caso a convulsão persistisse depois de re-administrado o sulfato de magnésio ( $3 \mathrm{~g}$ suplementares), e assim esta freqüência pode não corresponder à realmente encontrada em pacientes com eclâmpsia.

De qualquer forma, são necessários ensaios clínicos randomizados, duplo-cegos, comparando eficácia e efeitos colaterais das diferentes dosagens de infusão do sulfato de magnésio, para que se possa afirmar a superioridade de um ou de outro esquema. Dados de estudos observacionais, não-controlados, são insuficientes para se chegar a este tipo de conclusão.

Destaca-se outrossim que, mesmo quando a crise convulsiva não cessa de imediato ou vem a recorrer após a administração do sulfato, há evidências suficientes para se concluir que o sulfato de magnésio pode ser novamente utilizado, uma 
vez que a maior parte das pacientes nesta situação não voltará a ter convulsões ${ }^{2,7,8,11-13}$, e a droga é inequivocamente mais segura que a fenitoína e o diazepam ${ }^{12,13,15}$. Nesse grupo, é factivel supor que niveis terapêuticos satisfatórios (4-7 mEq/1) do sulfato de magnésio ${ }^{7,8}$ não tenham sido obtidos, talvez por fatores intrínsecos da paciente (má distribuição de líquidos, edema excessivo, índice de massa corpórea aumentado) ${ }^{2,7,8,17}$. Justifica-se assim repetir metade da dose de ataque e, cedendo a convulsão, aumentar a infusão de $1 \mathrm{~g}$ para 2 g/hora ${ }^{13,16}$.

Não encontramos nenhuma complicação associada à repetição da dose de sulfato de magnésio, o que também já foi descrito por outros autores ${ }^{7,9,12,15,19,20,22}$. Os resultados do "Eclampsia Trial", inclusive, mostram uma mortalidade materna significativamente menor entre as pacientes que receberam sulfato de magnésio em relação àquelas a quem se administrou fenitoína ou diazepam ${ }^{15}$. Ressaltamos ainda a vantagem de que o sulfato de magnésio, ao contrário do diazepam, não altera o estado de consciência materna e portanto não interfere no diagnóstico do coma, que pode ser uma complicação da eclâmpsia. No caso da fenitoína, esta última é mais tóxica e requer monitorização mais intensa, com efeitos colaterais mais significativos que o sulfato de magnésio ${ }^{12,13,15}$.

Além de obter a taxa de recorrência e avaliar os resultados da terapêutica, tentamos neste estudo identificar características clínicas e laboratoriais das pacientes que pudessem explicar a persistência das convulsões após o uso do sulfato de magnésio, de forma a identificar um subgrupo com risco aumentado de recorrência. Quando comparamos as pacientes com eclâmpsia de acordo com a presença ou não de recorrência, observamos que ambos os grupos eram similares em relação a idade (em torno de 25 anos), paridade (predominando as primiparas), idade gestacional (de aproximadamente 35 semanas) e niveis tensionais na admissão (em torno de 170 x $115 \mathrm{mmHg}$ ). A presença de hipertensão arterial sistêmica, no entanto, foi significativamente maior entre as pacientes que apresentaram recorrência, possivelmente porque o risco de hemorragia cerebral se sobreleva na hipertensão agravada pela gravidez, principalmente na eclâmpsia sobreposta ${ }^{2,6,8,20}$. Por outro lado, analisando-se os valores dos exames laboratoriais, os niveis de uréia, creatinina e ácido úrico foram maiores no grupo com recorrência, porém apenas a diferença em relação ao ácido úrico foi significativa $(7,2 \mathrm{mg} \%$ versus $6,1 \mathrm{mg} \%$ ). A proteinúria de fita (em torno de 4+) também não apresentou diferença significante entre os dois grupos. Esse achado pode ser explicado pelo fato de o ácido úrico encontrar-se mais elevado nas formas graves da hipertensão induzida pela gestação, podendo associar-se a pior prognóstico materno ${ }^{28,29}$ e portanto a maior risco de recorrência.

A recorrência parece constituir um marcador de gravidade, quer por refletir um sério processo já instalado, como a hemorragia cerebral $^{2,17,26}$, quer porque, na repetição das crises, advêm outras complicações igualmente sérias, como o coma e a acidose metabólica persistente ${ }^{2}$. Encontramos em nossa casuística uma maior freqüência não somente destas complicações como também de insuficiência renal aguda, edema agudo de pulmão e sindrome HELLP. Embora estas últimas não tenham apresentado diferença estatisticamente significativa, acreditamos que isso se deva tão somente ao pequeno número de pacientes com recorrência na amostra, o que aumenta a possibilidade de erro estatístico tipo II, uma vez que sua ocorrência foi bem mais freqüente no grupo com recorrência.

Tentando dirimir essas dúvidas e esclarecer alguns outros pontos obscuros, especialmente em relação ao perfil das pacientes com maior risco de recorrência, que poderiam talvez beneficiar-se de doses mais elevadas de manutenção do sulfato de magnésio, é nossa intenção prosseguir este estudo. A intenção é incorporar um número maior de pacientes para estudar com maior ênfase os fatores de risco associados à repetição da crise convulsiva após sulfato de magnésio. Deverão ser avaliadas variáveis que ainda não foram pesquisadas, como o índice de massa corpórea, o tempo de evolução da doença, a intensidade do edema e o estado hemodinâmico das pacientes, além das variáveis que já estudamos, porque com o aumento do tamanho da população diminuiremos as chances de erro tipo II. Análise de regressão logística múltipla poderá então ser efetuada para determinar o grupo de variáveis associados a maior risco de recorrência.

\section{SUMMARY}

Purpose: to determine the frequency of recurrence of seizures after anticonvulsant therapy with magnesium sulfate and to evaluate treatment and maternal prognosis.

Patients and Methods: a prospective cohort study was conducted, enrolling all cases of eclampsia managed at IMIP between January/1995 and June/1998. Magnesium sulfate and oxygen therapy were administered routinely and interruption of pregnancy was performed after maternal stabilization. The frequency of recurrence of seizures and its association with maternal complications were determined. $\chi^{2}$ test for association was used at a $5 \%$ level of significance. Results: twelve cases presented recurrence of convulsions 
after magnesium sulfate (10\%) and all received a repeated dose. In four of them convulsions persisted and they received intravenous diazepam. After diazepam, one patient still had seizures, with unsuccessful administration of phenytoin and therefore barbituric coma was induced (thionembutal). This patient had a CT-scan with evidence of intracerebral hemorrhage. Maternal complications were significantly more frequent in the group with recurrence: coma (16.7\% versus $0.95)$, acidosis (50\% versus $2.9 \%)$, pulmonary edema $(16.7 \%$ versus $2.9 \%$ ), cerebral hemorrhage $(16.7 \%$ versus $0 \%)$ and acute renal failure (16.7\% versus $1.9 \%)$. Three cases of maternal death occurred in patients with recurrence (25\%) versus 2 cases in patients without recurrence (1.9\%).

Conclusions: rate of recurrence after anticonvulsant therapy with magnesium sulfate is low (10\%) but it is associated with increased maternal morbidity and mortality. These cases must be managed in an intensive care unit and submitted to routine CT-scan because cerebral hemorrhage can be the cause of recurrence.

KEY WORDS: Eclampsia. Magnesium sulfate. Eclampsia: seizure recurrence. Maternal death.

\section{Referências}

1. Braga, LFCO, Nazareno, ER, Fanini ML, Soares VMN, Hirata VM. Relatório dos comitês de morte materna do Paraná. Femina 1993; 2:794-811.

2. Kahhale S, Zugaib M. Sindromes hipertensivas na gravidez. Rio de Janeiro: Atheneu; 1995. p.11540.

3. Moller B, Lindmark G. Eclampsia in Sweden, 19761980. Acta Obstet Gynecol Scand 1988; 65:30714.

4. Saftlas AF, Olson DR, Franks AL, Atrash HK, Pokras R. Epidemiology of preeclampsia and eclampsia in the United States 1979-1986. Am J Obstet Gynecol 1990; 163:461-5.

5. Sibai BM, Abdella TN, Spinnato JA, Anderson GD. Eclampsia. V. The incidence of non-preventable eclampsia. Am J Obstet Gynecol 1986; 154:581-6.

6. Sibai BM. Eclampsia. VI. Maternal-perinatal outcome in 254 consecutive cases. Am J Obstet Gynecol 1990; 163:1049-54.

7. Sibai BM. Magnesium sulfate is the ideal anticonvulsant in preeclampsia-eclampsia. Am J Obstet Gynecol 1990; 162:1141-5.

8. Cunningham FG, MacDonald PC, Gant NF, Leveno KJ, Gilstrap LC, Hankins GDV, et al. Williams obstetrics. 20 ${ }^{\text {th }}$ ed. Norwalk: Appleton \& Lange; 1997. p.693-744.

9. National High Blood Pressure Working Group Report on high blood pressure in pregnancy. Am J Obstet Gynecol 1990; 163:1691-712.

10.Pritchard JA, Cunningham FG, Pritchard SA. The Parkland Memorial Hospital protocol for treatment of eclampsia: evaluation of 245 cases. Am J Obstet Gynecol 1984; 148:951-63.
11.Usta IM, Sibai BM. Conduta de emergência nos casos de eclâmpsia puerperal. Clin Obstet Gynecol North Am 1995; 2:323-44.

12.Chien PF, Khan KS, Arnott N. Magnesium sulphate in the treatment of eclampsia and pre-eclampsia: an overview of the evidence from randomised trials. Br J Obstet Gynaecol 1996; 103:1085-91.

13.Witlin AG, Sibai BM. Magnesium sulfate therapy in preeclampsia and eclampsia. Obstet Gynecol 1998; 92:883-9.

14.Donaldson JO. Neurologic emergencies in pregnancy. Obstet Gynecol Clin North Am 1991; 18:199-212.

15. Which anticonvulsant for women with eclampsia? Evidence from the Collaborative Eclampsia Trial. Lancet 1995; 345: 1455-63.

16. Sibai BM. Magnesium sulfate in preeclampsiaeclampsia. Contemp Obstet Gynecol 1986; 29:155-9.

17.Barton JR, Sibai BM. Cerebral pathology in eclampsia. Clin Perinatol 1991; 18:891-910.

18.Sibai BM, Spinnato JA, Watson DL, Lewis JA, Anderson GD. Effects of magnesium sulfate on electroencephalographic findings in preeclampsia-eclampsia. Obstet Gynecol 1984; 64:261-6.

19.Barros ACSD, Kahhale S, Bittar RE, Carrara W, Cardoso MA, Zugaib M. Orientação terapêutica global da eclâmpsia: resultados da aplicação de um protocolo de tratamento padronizado. Rev Ginecol Obstet $1991 ; 2: 73-8$.

20.Frangieh AY, Friedman SA, Audibert F, Usta I, Sibai BM. Maternal outcome in women with eclampsia. Am J Obstet Gynecol 1996; 174:453.

21.Santos LC, Carvalho MMRA, Guerra GL, Porto AMF. Eclâmpsia: resultados maternos e perinatais. Rev IMIP 1993; 7:83-6.

22.Zuspan FP. Treatment of severe preeclampsia and eclampsia. Clin Obstet Gynecol 1966; 9:954-72.

23.Santos LC, Porto AMF, Carvalho MMRA, Guimarães VB. Obstetrícia: diagnóstico e tratamento. $1^{\mathrm{a}}$ ed. Rio de Janeiro: MEDSI; 1998. p.355-373.

24.Davey DA, MacGillivray I. The classification and definition of the hypertensive disorders of pregnancy. Am J Obstet Gynecol 1988; 158:892-8.

25. Sibai BM. The HELLP syndrome (hemolysis, elevated liver enzymes and low platelets): much ado about nothing? Am J Obstet Gynecol 1990; 162:311-6.

26.Thomas SV. Neurological aspects of eclampsia. J Neurol Sci 1998; 155:37-43.

27.Sharf B, Sharf M, Gonen R. Eclampsia. Handb Clin Neurol 1980; 39:537-44.

28.Zugaib M, Kahhale S, Barros ACSD, Miyada TLA, Camargo Jr HSA, Neme B. O papel da uricemia no diagnóstico e prognóstico da síndrome hipertensiva na gestação: estudo de 343 casos. Ginecol Obstet Bras 1985; 8:47-51.

29.Garrone C, Broso P. Uric acid and pre-eclampsia. Minerva Ginecol 1997; 49:213-6. 